\title{
$\operatorname{arCOS} D E S I G N$
}

\section{A incidência de disfunção lombar pélvica em fisioterapeutas}

Vânia Maria Batalha Cardoso (UFAM, Brasil)

vania_batalha@yahoo.com.br

Avenida General Rodrigo Otávio Jordão Ramos, 3.000

Coroado, Manaus, AM, Brasil CEP: 69077-000

Vanessa Caroline Santana Martinazzo (UFSC, Brasil)

m.vanessa@yahoo.com.br

Eugênio Andrés Diaz Merino (UFSC, Brasil)

eugenio.merino@ufsc.br

Giselle Schmidt Alves Diaz Merino (UFSC, Brasil)

gisellemerino@gmail.com 


\title{
A incidência de disfunção lombar pélvica em fisioterapeutas
}

Resumo: Os distúrbios osteomusculares relacionados ao trabalho (DORTs) ou lesões por esforços repetitivos (LER), são um conjunto de afecções de origem ocupacional que podem acometer fisioterapeutas.Os principais fatores de risco são de transferência de pacientes, posturas estáticas e terapia manual,tais profissionais são os mais acometidos por dores e lesões,durante o atendimento de pacientes, assumem posturas desconfortáveis resultando principalmente em comprometimento da região lombar. Embora o fisioterapeuta seja conhecedor de lesões observa-se a falta de autocuidado o que torna a prevenção importante necessidade,além de mais pesquisas e intervenções. O artigo apresenta problemas ergonômicos relacionados as atividades dos fisioterapeutas por meio de breve revisão de literatura, o conceito de ergonomia e sua aplicação para chegar a dados referentes as disfunções lombaresque poderão contribuir para futuras pesquisas e intervenções ergonômicas.

Palavras-chave: procedimentos de fisioterapia, lombalgia e ergonomia.

\section{The impact of lumbar pelvic's dysfunction in physical therapists}

\begin{abstract}
The work-related musculoskeletal disorders (MSDS) and repetitive strain injuries (RSI) are a group of diseases resulting from occupational hazards that can affect physiotherapists. The main risk factors are transferring patients, static postures and manual therapy, such people are the most affected by pain and injuries during the care of patients, assume awkward postures resulting in major impairment of the lumbar region. Lack of self-care which makes the important need prevention, and more research and interventions although the therapist is knowledgeable of lesions observed. The article presents ergonomic problems related activities of physiotherapists through brief literature review, the concept of ergonomics and their application to get data lumbar dysfunction that may contribute to future research and ergonomic interventions.
\end{abstract}

Key words: physiotherapeutic procedures, back pain and ergonomics. 


\section{Contextualização}

Entende-se que o trabalho subsiste na vida do homem como fator primordial na existência social e que os riscos ocupacionais podem causar vários danos à saúde do trabalhador.

Os padrões culturais e o estilo de vida moderna da população, impondo cada vezmais atividades especializadas e limitadas, provocam sobrecargas estruturais no corpo humano. A alta incidência de problemas posturais em adultos relaciona-secom a tendência para esse padrão de atividade, especializado ou repetitivo, aliadoao sedentarismo e vícios posturais carregados desde a infância (KENDALL, 1995).

Pode-se observar, na literatura, que há um crescimento quanto ao número de trabalhadores das mais diversas áreas profissionais que apresentam comprometimentos posturais, muitas vezes gerando dores na coluna vertebral, em consequência da sua jornada de trabalho.

Os profissionais da área da saúde estão entre aqueles com altos índices de dor na coluna vertebral relacionados à ocupação laboral.

Considerando tal premissa muitos profissionais, em especial os fisioterapeutas (objeto deste trabalho), são acometidos por problemas ocupacionais, principalmente as disfunções lombares decorrentes a posturasprejudiciais assumidas durante o atendimento dos pacientes. Buscar qualidade de vida no trabalhopara esses profissionaisé necessário e importante uma vez que além das posturasassumidas, realizam movimentos repetitivos com as mãos. Ressalta-se que estes se constituem nos principais fatores de doenças ocupacionais entre os fisioterapeutas.

De acordo Dolan (2006), a incidência das doenças ocupacionais vem crescendo gradativamente nos últimos anos, isso devido às inúmeras afecções e pelo grande estresse vivido nos ambientes de trabalho, onde a produtividade é o objetivo e as longas horas de trabalho são refletidas na saúde do homem. É importante salientar este assunto, pois são essas doenças as causadoras de afastamento e insatisfação no trabalho.

Neste contexto, os fisioterapeutas, que tem em seu trabalho o foco e atenção voltados para o tratamento de distúrbios em sistemas do corpo humano, cuidando de doenças ocupacionais, e buscando a recuperação de movimentos, muitas vezes contribuindo com a manutenção da agilidade e viabilizando, em alguns casos, o retorno ao desenvolvimento de atividades cotidianas das quaiso paciente tenha ficado impossibilitado de realizar em decorrência de traumas ou por doenças adquiridas.

Segundo Scholey e Hair (1989), o fato dos fisioterapeutas trabalharem emserviços de reabilitação com pacientes altamente dependentes e de 
sobrecargafísica, induz a uma efetiva participação dessa classe profissional, em um grupo de alto risco de comprometimentosmusculoesqueléticos desde o início de sua carreira profissional.

A identificação das causas da prevalência de algias na coluna vertebral,referentes as atividades laborais do fisioterapeuta, pode constituirse em importante dado para pesquisas e contribuições ergonômicas, já que esta última tem por meta melhorar as condições de trabalho visando o conforto a segurança e facilidade quanto a realização de operações. Neste aspecto a Ergonomia pode apresentar-se como alternativa para identificar dificuldades relacionadas as estas condições prejudiciais e convertê-las em requisitos e parâmetros ergonômicos que viabilizem a conscientização dos trabalhadores ou para gerar projetos ergonômicos. Para Iida(2005), o trabalhador que atua numa postura na qual se sente confortável e sem dor, apresentará melhores índices deeficiência e produtividade. Com posse desta realidade e a partir da Ergonomia pode-se utilizar tais dados para intervenções futuras e contribuir de formas mais efetiva para melhorar a qualidade de vida, a segurança e o conforto dos fisioterapeutas durante a realização de suas tarefas.

\section{Problemas}

Quando se afirma que "toda pesquisa tem início com algum tipo de problema, torna-se conveniente esclarecer o significado deste termo". Para Gil, (2010),"Uma acepção indica problemacomo mal-estar, sofrimento ou constrangimentos", o autor afirma ainda: "Com tudo na acepção científica o problema é qualquer questão não resolvida e que é objeto de discussão, em qualquer domínio do conhecimento". Pode-se entender que a identificação dos mesmos constitui-se em importante procedimento norteador para a construção dos resultados desejados em um projeto. Neste sentido, considerando o fisioterapeuta como foco deste trabalho, faz-se necessário apresentar neste item o objetivo maior da profissão para se chegar aos problemas que nortearão a presente pesquisa.

Segundo o Conselho Federal de Fisioterapia e Terapia Ocupacional - Coffito, a Fisioterapia é uma ciência que estuda, previne e trata os distúrbios cinéticos funcionais intercorrentes em órgãos e sistemas do corpo humano, gerados por alterações genéticas, por traumas e por doenças adquiridas (...).

Apesar da Fisioterapia ser uma profissão cujo objetivo maior é promover a saúde do indivíduo nos ambientes de trabalho e a prevenção e manutenção do sistema humano, suas condições de trabalho são precárias, o que proporciona a execução de tarefas que induzem danos à sua própria condição física 
no atendimento a seus pacientes.A incidência de disfunção lombar pélvica em fisioterapeutas, segundo Silva et al (2008), ocorre mais frequentemente na área de saúde, devido à postura física assumida pelos trabalhadores.

Dentre os profissionais da área de saúde, que apresentam distúrbios posturais,estão os fisioterapeutas, cuja atividade profissional implica em exigências do sistemamúsculo-esquelético, com movimentos repetitivos de membros superiores,manutenção de posturas estáticas e dinâmicas por tempo prolongado, e movimentosde sobrecarga para a coluna vertebral (PERES, 2002).

Dados epidemiológicos referentes a doença músculo esqueléticas(DME) em fisioterapeutas não são conhecidos no Brasil, os Conselhos Federal e os Regionais de Fisioterapia e o Terapia Ocupacional obtidos sobre a profissão mostra a inexistência de dados epidemiológicos sobre problemas lombares em fisioterapeutas; entretanto dirigentes de tais instituições apresentam preocupação uma vez que observa-se inúmeros caso de distúrbios músculo esqueléticos em fisioterapeutas mais não sabem precisar o quanto (TEDESCH, 2005).

É comum o desconforto lombar decorrente da improvisação durante o atendimento aos pacientes, quando posturas prejudiciais são adotadas paraa realização dos procedimentos. Essas posturas e movimentos resultam, em curto prazo, em dores que se prolongam além do horário de trabalho e, em longo prazo, podem resultar lesões permanentes, bem como deformidades.

Apesar da relevância do assunto, percebe-se a carência de dados referentes a sobrecargas posturais em fisioterapeutas, por atividades profissionais. Mas este é um assunto de grande importância no meio profissional, sendo necessário o apontamento dessas dificuldades para contribuição futuras tanto em pesquisa como intervenções em ergonomia.

\section{Objetivo}

Identificar as causas da prevalência de algias na coluna vertebral na atividade laboral do fisioterapeuta.

\section{Procedimentos metodológicos}

A pesquisa se apresenta conforme padrões de metodologia da pesquisa e se caracteriza como bibliográfica, pois foram selecionados materiais em artigos e livros referente as atividades dos fisioterapeuta e suas dificuldades 
decorrente das posturas adotadas nos procedimentos terapêuticos,além deste material recorreu-se aoconceito de ergonomia, foram considerados relatos e observações de profissionais em atendimento à pacientes.

Foram realizadas observações assistemáticas com fisioterapeutas durante seu trabalho o que possibilitou alguns registros fotográficos e relatos, as informações são significativas, pois confirmam o que é apresentado na literatura atual.

\section{Fisioterapia e trabalho}

A fisioterapia é a ciência da saúde que estuda, previne e trata distúrbios cinéticos funcionais intercorrentes em órgãos e sistemas do corpo humano, gerados por alterações genéticas por traumas e por doenças adquiridas. Suas ações são fundamentadas em mecanismos próprios (Coffito, 1984).O mesmo Conselho define o fisioterapeuta como profissional de Saúde, com formação acadêmica Superior, habilitado à construção do diagnóstico dos distúrbios cinéticos funcionais (Diagnóstico Cinesiológico Funcional), a prescrição das condutas fisioterapêuticas, a sua ordenação e indução no paciente bem como, o acompanhamento da evolução do quadro clínico funcional e as condições para alta do serviço.

Ressalta-se que os fisioterapeutas, passam boa parte do seu dia em atendimentos, que geram desconforto lombar, e que atuam grande parte do seu tempo no ambiente natural da clínica de fisioterapia e hospitais com carga horária de 8 horas semanais sujeitos a constrangimentos posturais bem como atividades repetitivas podendo ocorrer distúrbios osteomusculares relacionados ao trabalho, comprometendo sua saúde, onde passa boa parte dedicandose a saúde do próximo, dados estes fundamentais para estudos ergonômicos e segurança do trabalho, áreas do saber que visam melhorar as condições de trabalho e proporcionar melhor qualidade de vida aos seres humanos.

Segundo relatado por profissionais da área,"as posturas mais comumente utilizadas pelos Fisioterapeutas são as posturas em pé, sentada, ajoelhada e semi-sentada, com flexão anterior do tronco e flexão com rotação do tronco, mantidas por tempo prolongado". Torén, (2001) afirma que, o tronco estando em rotação na atividade de trabalho sobrecarrega as articulações da coluna vertebral e produz a sensação de desconforto e dor para essa região.

Cromie (2000), refere alguns fatores pessoais relacionados aos distúrbios posturais em fisioterapeutas como: trabalhar próximo ao limite físico continuar trabalhando mesmo lesionado, falta de treinamento ou treinamento inadequado na prevenção destes distúrbios. 
As doenças no trabalho são provocadas pelo uso inadequado e excessivo do sistema que agrupa ossos, músculos, nervos e tendões. Essas afecções típicas de trabalho intenso e repetitivo são causadas por diversas pressões existentes no trabalho, tanto físicas como psíquica (RAGASSON, 2004).

Segundo a definição dada pela Organização Mundial da Saúde (OMS), a promoção da saúde consiste em todas as medidas que permitem a indivíduos, grupos e instituições exercerem o maior controle sobre os fatores que afetam a saúde no intuito de proporcionar saúde a estes (DOLAN, 2006).

Fora do ambiente de trabalho, deve-se dar continuidade ao tratamento, pois os tempos modernos contribuem para o surgimento de desequilíbrios emocionais, como o estresse, que resulta em má postura, geralmente expressa por um aumento de tensão na região cervical e lombar.

Observa-se que grande parte das agressões à coluna vertebral em trabalhadores da saúde está relacionada a condições ergonômicas inadequadas de mobiliários, postos de trabalho e equipamentos utilizados nas atividades cotidianas.A intervenção ergonômica ao considerar restrições financeiras pode acontecer através de ações simples de conscientização, mudanças em layoutse recorrer a materiais e produtos existentes ou alternativos (pertinente a função requerida) para solucionar os problemas identificados. Quanto a Ergonomia, estima-se que a adoção de medidas ergonômicas de baixo custo no ambiente de trabalho é capaz de reduzir cerca de $80 \%$ a incidência de dores lombares (couto,2002). Conhecer e compreender as dificuldades desses profissionais nos trará um avanço para contribuir com os dados que ainda não estão oficializados e desenvolver pesquisa e proposta que permitam melhorar sua qualidade de vida.

A ergonomia tem sido difundida como uma das mais importantes estratégias para reduzir os problemas originados por situações de trabalho que causam doenças no sistema músculo-esquelético. Considera-se, então, de primordial importância, que seja divulgada entre os trabalhadores com o objetivo de criar hábitos para o desenvolvimento de uma consciência critica em relação aos efeitos do ambiente de trabalho relacionados à saúde.

Alguns fisioterapeutas preconizam que a saúde física e as emoções das pessoas dependem quase que exclusivamente da sua interpretação do mundo exterior. A realidade de cada pessoa é o produto de sua própria criação. E, quanto mais 'ela' entende as pressões e situações que lhe influenciam, melhor 'ela' se adapta às suas demandas. Por isso, há necessidade daconscientização dos dados levantados nesta pesquisa para melhores condições de trabalho e adequação dos equipamentos, ambiente e preservação da sua saúde. Assim, o binômio qualidade de vida e produtividade pode ser melhorado. 


\section{Fatores de risco para fisioterapeuta}

Os três fatores de riscos primários associados ás LER/DORTS, são movimentos repetitivos, posturas inadequadas e níveis altos de força.A maioriados fisioterapeutas atribui seus primeiros sintomas ao processo de trabalho que requer o desempenho de inúmeras tarefas relacionadas aos cuidados dos pacientes e clientes, incluindo atividades como agachamento, treino de marcha, resistência manual, flexão e ou rotação do tronco e adoção de posturas muitas vezes inadequadas (figura 01e 02). Entre as atividades observadas como responsáveis pelo inicio dos sintomas, entre os terapeutas, ressalta-se a transferência de pacientes dependentes comosendo responsável por grande número de profissionais acometidos. Outras atividades com potencial lesivo são a terapia manual (figura01-04), posturas estáticas por longo tempo, flexão e rotação do tronco com ou sem sobrecarga de peso associada e levantamento de peso (figura 03).

Hignett enfatiza o princípio ergonômico de "adequação do trabalho ao trabalhador", e não ao contrario muitas vezes, porem, o trabalho com seres humanos não permite a aplicação de tão conceito, devido a diferenças antropométricas individuais dos pacientes de acordo com o autor, difunde-se que o paciente deva vir sempre em primeiro lugar é um dos fatores responsáveis pela negligencia na adoção de posturas e técnicas a serem realizadas. Portanto há necessidadede reconhecer a vulnerabilidade da profissão e a seleção de técnicas mais adequadas durante o trabalho de fisioterapeutas.

Registros de fatores de riscos que comuns entre os fisioterapeutas tais como: são movimentos repetitivos, posturas inadequadas e níveis altos de força.

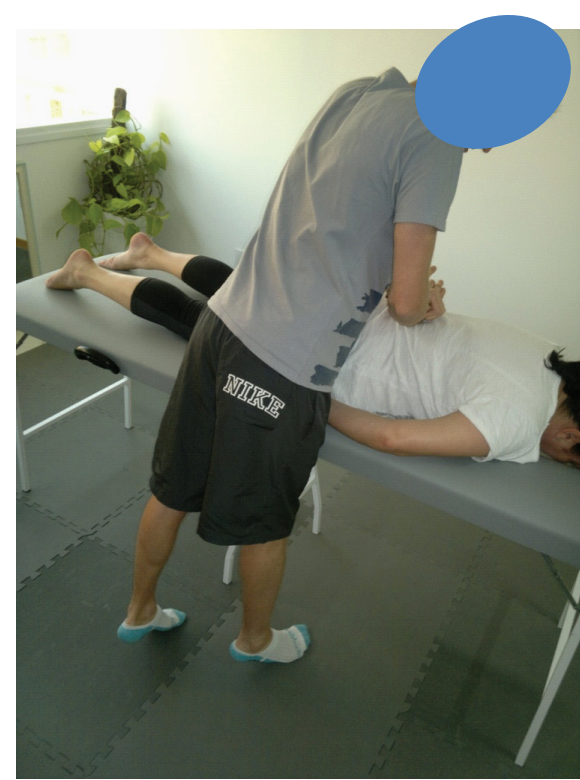

Figura 01. Plantiflexão pés manipulação com sobrecarga

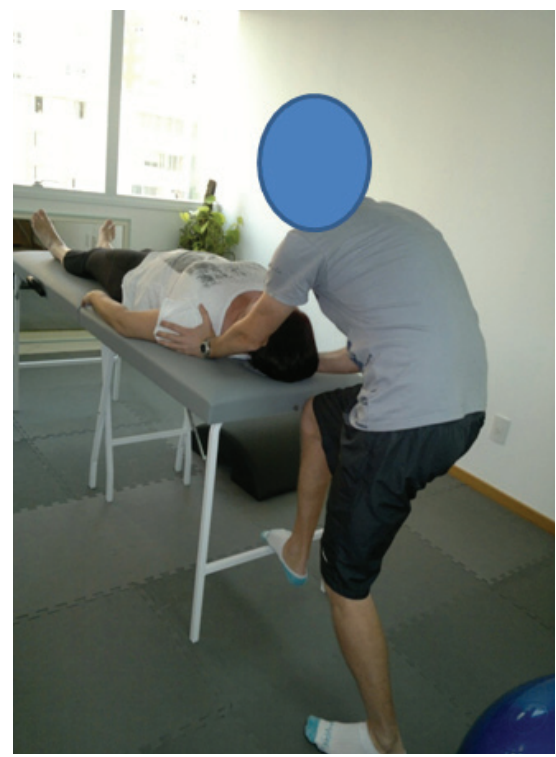

Figura 02. Flexão da coluna lombar 


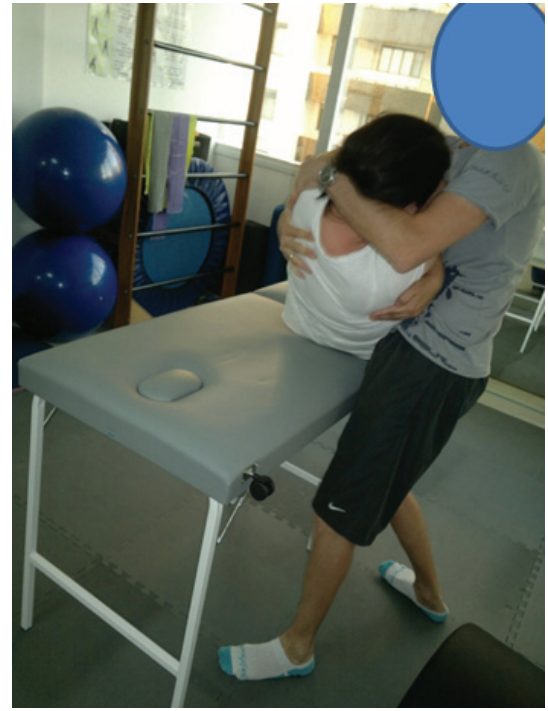

Figura 03. Sobrecarga

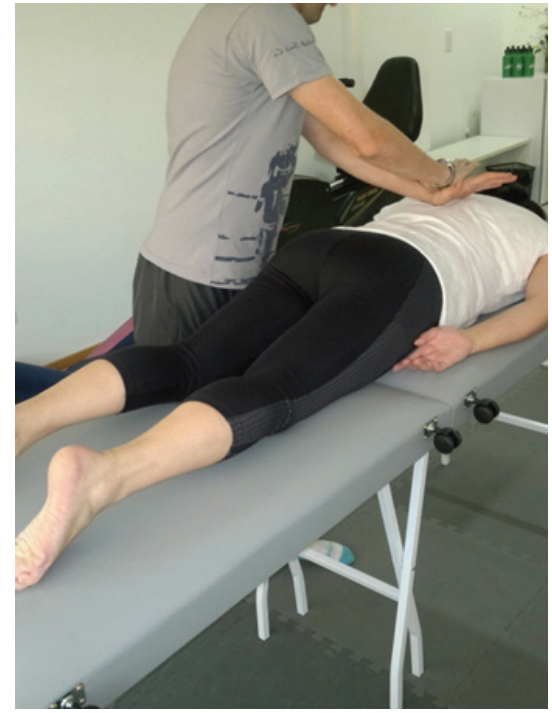

Figura 04. Uso intenso de mãos e aplicação de força

\section{Ergonomia}

Ergonomia ou fatores humanos é a disciplina cientifica que trata da compreensão das interações entre os ser humanos e outros elementos de um sistema, é a profissão que aplica teorias princípios, dados e métodos, a projetos que visam otimizar o bem estar humano e a performance global dos sistemas (IEA, 2000).

Cabe aqui a definição de Ergonomia como ciência e prática, pois para Moraes e Mont’Alvão (2009), "A única e específica tecnologia da Ergonomia é a tecnologia da Interface homem-sistema. A Ergonomia como ciência trata de desenvolver conhecimentos sobre as capacidades, limites e outras características do desempenho humano e que se relacionam com projeto de interfaces, entre indivíduos e outros componentes do sistema. Como prática, a Ergonomia compreende a aplicação da tecnologia da interface homem-sistema a projeto ou modificações de sistemas para aumentar a segurança, conforto e eficiência do sistema e da qualidade de vida".

Em geral, os domínios da ergonomia são: Física, Cognitiva, Organizacional. Considerando que o foco deste seja o fisioterapeuta no ambiente de trabalho, o interesse principal recai sobre a Ergonomia física que está relacionada com as características da anatomia humana, antropometria, fisiologia e biomecânica em sua relação a atividade física com relevância aos estudos da postura no trabalho, manuseio de materiais, movimentos repetitivos, distúrbios músculo-esqueletais em relação ao trabalho, postos de trabalho além de segurança, saúde e conforto.

Pinheiro e França (2006) consideram a ergonomia do trabalho é um recurso de prevenção que depende da participação de todos desde a empresa, cargos de chefia, passando pelos trabalhadores e profissionais da saúde até a 
Comissão Interna de Prevenção de Acidentes - CIPA. A ergonomia aplicada como agente de prevenção para lesões e doenças ocupacionais é um avaliador fundamental. "A Ergonomia do trabalho tem por objetivo avaliar e identificar os fatores de riscos nos postos de trabalho, ou seja, verificar os movimentos bruscos e repetitivos, uso da força, posições forçadas, tempo prolongado, aspectos organizacionais e aspectos psicossociais" (PINHEIRO; FRANÇA, 2006).

Reconhecendo que as pesquisas ergonômicas podem propiciar melhorias nas condições de trabalho, e elevar a qualidade dos serviços prestados por uma instituição, esta pesquisa objetivou identificar, a incidência de dores na coluna destes profissionais, que atuam na área de reabilitação para disponibilizar material para utilização em novas pesquisas e futuras intervenções ergonômicas.

Segundo (Hendrick,2003) "Há muito que os profissionais da ergonomia reconheceram o enorme potencial da nossa disciplina para melhorar a saúde,segurança e conforto das pessoas além da produtividade humana e do sistema. Certamente,através da aplicação da tecnologia única da interface humano-sistema, nós temos o potencial de realmente mudar a qualidade de vida de praticamente todas as populações do globo".

\section{Considerações finais}

O presente artigo enfatiza a necessidade de maiores cuidados por parte dos fisioterapeutas com sua saúde no exercício de suas atividades laborais em relação a sua qualidade de vida bem como a manutenção de sua saúde principalmente em relação a problemas relacionados a coluna vertebral.

Como exposto, estes profissionais, trabalham em clínicas, hospitais e consultórios assumindo posturas prejudiciais que são necessárias ao exercício de sua profissão. São muitos os Fisioterapeutas que exercem suas atividades ematendimento ao paciente neurológico em colchonetes ou tatames, dispostos no chãoou sobre um tablado de madeira, onde se posicionam de maneira desconfortável ede modo desajeitado, realizando flexão, com ou sem rotação de tronco, e muitas vezes tendo que realizar movimentos de sustentação de peso ou vencer algum graude hipertonia do paciente, respondendo a movimentos súbitos ou inesperados dopaciente, utilizando sua força corporal numa posição indesejável e com tarefasrepetitivas. Normalmente, essa postura sentada é acompanhada de flexão dosmembros inferiores, o que torna o trabalho bastante exaustivo, tendo poucas opções demudanças de postura.

Dor nas costas é uma das desordens ocupacionais mais apontadas pelos pesquisadores, sendo decorrente de posturas inadequadas no ambiente de trabalho, segundo Couto (1995), é identificada por estudos epidemiológicos 
e análises biomecânicas. A adoção de posturas inadequadas na realização de determinadas funções,associadas a outros fatores de risco existentes no posto de trabalho, comosobrecarga imposta à coluna vertebral, vibrações e manutenção de uma postura portempo prolongado constitui "nas maiores causas de afastamento do trabalho e de sofrimento humano".

Buscar qualidade de vida no trabalho para esses profissionais é necessário e importante uma vez que os movimentos repetitivos das mãos e as posturas necessárias a realização do trabalho do fisioterapeuta, durante o atendimento aos pacientes,comprometem sua saúde. Apesar de estes representaremos principais fatores de doenças ocupacionais entre fisioterapeutas, os problemas se repetem e pouco tem sido feito para a redução das lesões, doenças e dificuldades ocupacionais vividas por este seguimento de profissionais. Pode-se considerar com base no exposto que através da Ergonomia e da usabilidade intervenções podem ocorrer associadas a conscientização e proposição de equipamentos mais viáveis e compatíveis as especificidades do trabalho e dos diferentes profissionais.

Para trabalhos futuros tal material constitui-se em importante fonte de consulta, mas fica advertida a necessidade de um maior levantamento de dados, continuidade deste trabalho e o diagnóstico de outras doenças ou lesões que podem acometer este profissional.

\section{Referências}

BRISSCHOP, P. Instabilidade lombar: implicações para o fisioterapeuta. Revista Terapia Manual: Fisioterapia Manipulativa, Londrina, v.1, n. 4, p. 122126, abr./jun., 2003.

CARREIRO R., TRELHA C., MASTELARI, H. Disturbios Osteomusculares do Trabalho em Fisioterapeutas: revisão de literatura. Revista de Fisioterapia e Pesquisa da USP, SãoPaulo, Vl. 13 n.1, p.53-59, jan./abril, 2006.

CHEREM,J.A.; MAGAJEWSKI,F. Ergonomia: o trabalho como sujeito, o trabalho como objeto. Episteme. Publicação Cientifica da Universidade do Sul de Santa Catarina, Tubarão,v. 10 n. 28/29,p.59-71, nov./jun. 2002/2003

COUTO, H. Ergonomia aplicada ao trabalho em 18 lições. Belo Horizonte: Ergo, 2002.

COFFITO, Decreto-Lei 938/69, Lei 6.316/75, Resoluções do COFFITO, 
Decreto 9.640/84, Lei 8.856/94. Definição deFisioterapia.

DOLAN, S.L. Estresse, auto-estima, saúde e trabalho. Tradução: J. Simões. Rio de janeiro: Qualitymark, 2006.

DUL, J.; WEERDMEESTER, B. Ergonomia Prática. São Paulo:Edgard Blucher,1995.

HENDRICK,W .A. Boa ergonomia é boa economia. ABERGO, 2003.

IIDA, I. Ergonomia - Projeto e produção. 2.ed. São Paulo: Edgard Blücher, 2005.

JORDAM,P. An intoduction usabilility. Taylor and Francis, London, 1998.

KENDALL, P.F.; McCREARY E. K.; PROVANCE P.G. Músculos Provas e funções. São Paulo: Manole, 1995.

MORAES, A. de, Mont'Alvão, C. Ergonomia Conceitos e Aplicações. 4. ed. Rio de Janeiro: iUsEr, 2009.

PERES, C. P. A., 2002. Estudo de Sobrecargas Posturais em Fisioterapeutas: Uma Abordagem Biomecânica Ocupacional. 2002., Dissertação (Mestrado em Engenharia de Produção), Programa de Engenharia de Produção UFSC, Florianópolis.

PINHEIRO, A. K. S.; FRANÇA, M. B. A. ERGONOMIA: aplicada á anatomia e a fisiologia do trabalhador. Goiânia: AB, 2006 v2.

RAGASSON, C. A. P. Qualidade no trabalho: estudo das condições de trabalho. Cascavel Paraná, Coluna do Saber, 2004.

SCHOLEY, M.; HAIR, M. Back Pain in Physiotherapists Involved in Back Care Education. Ergonomics, 38 (2): p. 179-190.1989.

SILVA, M. F.; SANTOS JUNIOR, F. F. U.; ARCANJO, G. N. Dor nas costas em estudantes do último ano de fisioterapia: um estudo de prevalência. Revista Terapia Manual: Fisioterapia Manipulativa, v. 6, n. 23, p. 48 - 53, 2008.

TEDESCHI M.A.,2005. Indicadores para a gestão de distúrbios 
Músculo-esqueléticosemfisioterapeutas.2005.Tese(DouradoemEngenharia de Produção e Sistemas) Programa de Engenharia de Produção UFSC, Florianópolis.

TORÉN, A. Applied Ergonomics, 32: p. 583-591. 2001. 
Recebido em: 10/06/2013

Aceito em: 01/10/2013

\section{Como citar}

CARDOSO, Vânia Maria Batalha; MARTINAZZO, Vanessa Caroline Santana; MERINO, Eugênio Andrés Diaz; MERINO, Giselle Schmidt Alves Diaz. A incidência de disfunção lombar pélvica em fisioterapeutas. Arcos Design. Rio de Janeiro: PPD ESDI - UERJ. Volume 7 Número 2 Dezembro 2013. pp. 80-93. Disponível em: [http://www.e-publicacoes.uerj.br/index.php/ arcosdesign]

\section{DOI}

10.12957/arcosdesign.2013.12183

\section{c) (i) (2)}

A Revista Arcos Design está licenciada sob uma licença Creative Commons Atribuição - Não Comercial - Compartilha Igual 3.0 Não Adaptada. 